\title{
MORE ON THE COUNTABILITY INDEX AND THE DENSITY INDEX OF $S(X)$
}

\author{
by K. D. MAGILL, Jr. \\ (Received 27th October 1988) \\ Dedicated to Professor Yukihiro Kodama on his 60th Birthday
}

\begin{abstract}
The countability index, $C(S)$, of a semigroup $S$ is the smallest integer $n$, if it exists, such that every countable subset of $S$ is contained in a subsemigroup with $n$ generators. If no such integer exists, define $C(S)=\infty$. The density index, $D(S)$, of a topological semigroup $S$ is the smallest integer $n$, if it exists, such that $S$ contains a dense subsemigroup with $n$ generators. If no such integer exists, define $D(S)=\infty . S(X)$ is the topological semigroup of all continuous selfmaps of the locally compact Hausdorff space $X$ where $S(X)$ is given the compact-open topology. Various results are obtained about $C(S(X))$ and $D(S(X))$ ). For example, if $X$ consists of a finite number $(>1)$ of components, each of which is a compact $N$-dimensional subspace of Euclidean $N$ space and has the internal extension property and $X$ is not the two point discrete space. Then $C(S(X))$ exceeds two but is finite. There are additional results for $C(S(X))$ and similar results for $D(S(X))$.
\end{abstract}

1980 Mathamatics subject classification (1985 Revision): 54H15.

\section{Introduction}

The countability index, $C(S)$, of a semigroup $S$ is the smallest integer, $N$, if such an integer exists, with the property that each countable subset of $S$ is contained in a subsemigroup with $N$ generators. If no such integer exists, we define $C(S)=\infty$. The density index, $D(S)$, of a topological semigroup $S$ is the smallest positive integer $N$, if such an integer exists, such that $S$ contains a dense subsemigroup with $N$ generators. If no such integer exists, we define $D(S)=\infty$ here as well. We will assume that all spaces discussed in this paper are Hausdorff and when we topologize $S(X)$, the semigroup of all continuous selfmaps of the topological space $X$, it will always be with the compact-open topology. It has long been known that if $X$ is locally compact, then $S(X)$ with the compact-open topology is a topological semigroup. The converse is not true without some restrictions on the spaces but recently $S$. Subbiah [6] has shown that the converse is true within a very extensive class of spaces which includes all completely regular spaces which contain an arc and all 0-dimensional spaces. That is for any one of these spaces, $S(X)$ is a topological semigroup if and only if $X$ is locally compact. It is in Section 3 that we regard $S(X)$ as a topological semigroup and we assume there that the spaces are actually compact. Cook and Ingram [1] and S. Subbiah [5] have shown that there are a considerable number of topological spaces, including all Euclidean $N$-cells, for which $C(S(X))=2$. Since $S(X)$ is separable for these spaces when given the compactopen topology, it readily follows that $D(S(X))=2$ also. In addition, we know that 
$C(S(X))=D(S(X))=\infty$ when $X$ is any one of the Euclidean $N$-spheres [3]. It is immediate that $C(S(X))=D(S(X))=1$ if $X$ is the one-point space and it is almost as immediate that this is the only case for which $C(S(X))=D(S(X))=1$. To see this, simply observe that the countability index of any noncommutative semigroup must exceed one and $S(X)$ is not commutative if $X$ has more than one point. If $D(S(X))=1$ then $S(X)$ has a commutative dense subsemigroup so that in this case also, $S(X)$ would have to be commutative. It is well known that $C(S(X))=D(S(X))=2$ if $X$ is the two point discrete space and $C(S(X))=D(S(X))=3$ if $X$ is a finite discrete space with at least three points. In this paper we show that there is quite an extensive class of spaces with the property that both $C(S(X))$ and $D(S(X))$ are both finite and both exceed two. It is still an open problem to determine precisely what these two numbers are in these cases.

\section{The countability index}

The following lemma is useful. Its proof is completely straightforward and will be omitted.

Lemma 2.1. Let $S$ and $T$ be two semigroups and suppose $S$ is a homomorphic image of T. Then $C(S) \leqq C(T)$.

Theorem 2.2. Let $X$ be a disconnected space with a finite number of components which is not the two point discrete space. Then $C(S(X)) \geqq 3$.

Proof. Suppose first that $X$ has only two components and denote them by $A$ and $B$ respectively. Since $X$ is not the two point discrete space, one of the components, say $A$, has more than one point and is therefore infinite. Choose a countably infinite collection $\left\{a_{n}\right\}_{n=1}^{\infty}$ of points from $A$ and any point $b \in B$. Define a continuous selfmap $\left\langle a_{n}, b\right\rangle$ of $S(X)$ by

$$
\begin{array}{lll}
\left\langle a_{n}, b\right\rangle(x)=a_{n} & \text { for } & x \in A \\
\left\langle a_{n}, b\right\rangle(x)=b & \text { for } & x \in B .
\end{array}
$$

Next, let $\langle b\rangle$ denote the constant map which carries everything into the point $b$. Now let $T$ consist of $\langle b\rangle$, the identity map $\delta$, and all the maps in the collection $\left\{\left\langle a_{n}, b\right\rangle\right\}_{n=1}^{\infty}$. Suppose $T \subseteq\langle\langle f, g\rangle\rangle$, where $\langle\langle f, g\rangle\rangle$ denotes the subsemigroup generated by the two continuous selfmaps $f$ and $g$. Denote the ranges of $f$ and $g$ by $\operatorname{Ran} f$ and $\operatorname{Ran} g$ respectively and suppose we have

$$
\operatorname{Ran} f \cap A \neq \varnothing \neq \operatorname{Ran} f \cap B
$$

and

$$
\text { Ran } g \cap A \neq \varnothing \neq \operatorname{Ran} g \cap B
$$


But this would imply that $\langle b\rangle \notin\langle\langle f, g\rangle\rangle$ so we must have either $\operatorname{Ran} f$ or $\operatorname{Ran} g$ contained entirely in $A$ or entirely in $B$. It turns out that we lose no generality if we assume that $\operatorname{Ran} f \subseteq A$. It follows that $f$ cannot possibly be a factor of any of the functions $\left\langle a_{n}, b\right\rangle$ and therefore, we must have $\left\{\left\langle a_{n}, b\right\rangle\right\}_{n=1}^{\infty} \subseteq\langle\langle g\rangle\rangle$. In a similar manner, we must also have $\delta \in\langle\langle g\rangle\rangle$. But this means $g^{m}=\delta$ for some positive integer $m$ which implies that $\langle\langle g\rangle\rangle$ is a finite cyclic semigroup. This, of course, is a contradiction and we have shown that $C(S(X)) \geqq 3$ whenever $X$ has two components.

Now consider the case where $X$ has $N \geqq 3$ components and denote them by $\left\{A_{n}\right\}_{n=1}^{N}$. Let $\mathscr{T}_{N}$ denote the semigroup of all functions on the set $\{1,2, \ldots, N\}$ and define a map $\phi$ from $S(X)$ onto $\mathscr{T}_{N}$ as follows:

$$
(\phi(f))(n)=m \quad \text { if and only if } f\left[A_{n}\right] \subseteq A_{m} .
$$

One can verify that $\phi$ is a homomorphism from $S(X)$ onto $\mathscr{T}_{N}$ and since it is well known that $C\left(\mathscr{T}_{N}\right)=3$, it follows from Lemma 2.1 that $C(S(X)) \geqq 3$ in this case also.

Some remarks. If, in the previous theorem, one does not rule out the two point discrete space then the conclusion is false since $C(S(X))=2$ when $X$ consists of two points. Furthermore, one cannot simply delete the requirement that $X$ have a finite number of components and hope to prove the theorem because it is known $[1,5]$ that $C(S(X))=2$ if $X$ is infinite and discrete, the space of rational numbers, the Cantor discontinuum or the space of irrational numbers.

Definition 2.3. A space $X$ has the internal extension property if each continuous map from a closed subset of $X$ into $X$ can be extended to a continuous selfmap of $X$.

Theorem 2.4. Let $X$ consist of a finite number of components, each of which is a compact $N$-dimensional subspace of Euclidean $N$-space and has the internal extension property. Then $C(S(X))$ is finite.

Proof. In this proof, we modify and use some of the techniques used in the proof of Theorem 4.2 of [5]. We cannot appeal directly to that theorem because the space under consideration there is required to have the internal extension property and our space $X$ definitely does not have it. Denote the components of $X$ by $\left\{C_{j}\right\}_{j=1}^{M}$. According to Theorem IV $3\left[2, \mathrm{p}\right.$. 44] each $C_{j}$ contains a nonempty subset which is open in $E^{N}$, the Euclidean $N$-space where $N$ is the dimension of $C_{j}$. Choose a subspace $K_{j}$ of that open subset which is homeomorphic to the Euclidean $N$-cell, $I^{N}$. It readily follows that each $K_{j}$ contains a countably infinite mutually disjoint family $\left\{D_{j, n}\right\}_{n=1}^{\infty}$ of subspaces each of which is homeomorphic to $C_{j}$ and moreover, $\left(c \ell D_{j}\right) \backslash D_{j}=\left\{p_{j}\right\}$ where $D_{j}=\cup\left\{D_{j, n}\right\}_{n=1}^{\infty}$. For each $n$, let $h_{n}$ be a homeomorphism from $B_{n}$ onto $X$ where $B_{n}=\cup\left\{D_{j, n}\right\}_{j=1}^{M}$. Now consider the mapping $g_{1}$ defined by $g_{1} \mid B_{n}=h_{n-1}^{-1} \circ h_{n}$ for $n>1$ and extend $g_{1}$ continuously over $c \ell \cup\left\{B_{n}\right\}_{n=2}^{\infty}$ by defining $g_{1}\left(p_{j}\right)=p_{j}$ for each $j$. At this point, $g_{1}$ maps a closed subset of $C_{j}$ into $K_{j}$ and for each $j$ can be extended to a continuous selfmap of $X$ since each $C_{j}$ has the internal extension property (this also follows from the fact that 
each $K_{j}$ is an absolute retract). The result is an extension to a continuous selfmap of $X$. The same argument results in the conclusion that there exists a continuous selfmap $g_{2}$ of $X$ such that $g_{2} \mid B_{n}=h_{n+1}^{-1} \circ h_{n}$. Because each $C_{j}$ has the internal extension property, $h_{1}$ can be extended to a continuous selfmap of $X$ which we denote by $g_{3}$ and we let $g_{4}=h_{1}^{-1}$. We need to define one more function but it depends upon the family which is to be contained in the subsemigroup which is generated so let $\mathscr{F}$ be a countable family of continuous selfmaps of $X$. Decompose the family of components of $X$ into a (necessarily) finite number of subcollections $\left\{\mathscr{C}_{j}\right\}_{j=1}^{K}$ and choose $K$ components of $X$. There is no loss of generality if we assume we actually have the first $K$ components $\left\{C_{j}\right\}_{j=1}^{K}$ as we originally ordered them and this will make subsequent notation considerably simpler. Next, let $\left\{f_{n}\right\}_{n=1}^{\infty}$ consist of all those functions in $\mathscr{F}$ which, for each $j$, maps each of the components in the family $\mathscr{C}_{j}$ into the component $C_{j}$. We have assumed that there are an infinite number of such functions since we have nothing to prove if there are not. Now we are in a position to define our fifth function $g_{5}$. First of all define $g_{5} \mid B_{n}=h_{n}^{-1} \circ f_{n} \circ h_{n}$ and continuously extend $g_{5}$ over $c \ell \cup\left\{B_{n}\right\}_{n=1}^{\infty}$ as follows. For $C_{i} \in \mathscr{C}_{j}$ define $g_{5}\left(p_{i}\right)=p_{j}$. Now the restriction of $g_{5}$ to of $D_{i}$ is a continuous map from a closed subset of $C_{i}$ into $K_{j} \subseteq C_{j}$ and since $K_{j}$ is an absolute retract, $g_{5}$ can be continuously extended so as to map all of $C_{i}$ into $C_{j}$. This holds for all $C_{i} \in \mathscr{C}_{j}$ and it holds in turn for all $j$. The result is a continuous selfmap $g_{5}$ of $X$ with the property that $g_{5} \mid B_{n}=h_{n}^{-1} \circ f_{n} \circ h_{n}$. One next verifies that $g_{1}^{n-1}(x)=h_{1}^{-1} \circ h_{n}(x)$ for $x \in B_{n}$ where $n>1$ and $g_{2}^{n-1}(x)=h_{n}^{-1} \circ h_{1}(x)$ for $x \in B_{1}$. With these two facts a routine verification yields the fact that

$$
f_{n}=g_{3} \circ g_{1}^{n-1} \circ g_{5} \circ g_{2}^{n-1} \circ g_{4}
$$

This proves that $\left\{f_{n}\right\}_{n=1}^{\infty} \subseteq\left\langle\left\langle g_{1}, g_{2}, g_{3}, g_{4}, g_{5}\right\rangle\right\rangle$ the subsemigroup generated by those five functions. Since the entire family $\mathscr{F}$ can be decomposed into a finite number of subfamilies like $\left\{f_{n}\right\}_{n=1}^{\infty}$, we conclude that $\mathscr{F}$ is contained in a finitely generated subsemigroup. In other words the countability index of $S(X)$ is finite.

Theorem 2.5. Let $X$ consist of two components, each of which is a compact $N$-dimensional subspace of Euclidean $N$-space and has the internal extension property. Then $3 \leqq C(S(X)) \leqq 8$ and if the two components are homeomorphic then $C(S(X)) \leqq 7$.

Proof. In view of Theorem 2.2 we need only show that $C(S(X)) \leqq 8$ in general and $\leqq 7$ when the components are homeomorphic. We consider the general case first. The functions $g_{1}, g_{2}, g_{3}$ and $g_{4}$ are defined just as in the proof of Theorem 2.4 where, in this case, $N=2$. Now let $\mathscr{F}$ be a countable family of continuous selfmaps of $S(X)$ and let $C_{1}$ and $C_{2}$ be the two components of $X$. Let $\mathscr{F}_{1}$ consist of all functions $f$ in $\mathscr{F}$ with the property that $f\left[C_{1}\right] \subseteq C_{1}$ and $f\left[C_{2}\right] \subseteq C_{2}$. Let $\mathscr{F}_{2}$ consist of all functions $f$ in $\mathscr{F}$ with the property that $f\left[C_{1}\right] \subseteq C_{2}$ and $f\left[C_{2}\right] \subseteq C_{1}$. Let $\mathscr{F}_{3}$ consist of all functions in $\mathscr{F}$ such that $f[X] \subseteq C_{1}$ and finally, let $\mathscr{F}_{4}$ consist of all functions $f$ in $\mathscr{F}$ such that $f[X] \subseteq C_{2}$. For each of the families $\mathscr{F}_{j}, 1 \leqq j \leqq 4$, one produces a function $h_{j}$ exactly as $g_{5}$ was obtained in the proof of Theorem 2.4 and it follows also as in the proof of that theorem that $\mathscr{F}_{j} \subseteq\left\langle\left\langle g_{1}, g_{2}, g_{3}, g_{4}, h_{j}\right\rangle\right\rangle$ for $1 \leqq j \leqq 4$. Thus $\mathscr{F} \subseteq\left\langle\left\langle g_{1}, g_{2}, g_{3}, g_{4}, h_{1}, h_{2}, h_{3}, h_{4}\right\rangle\right\rangle$ and we see that $C(S(X)) \leqq 8$. 
Now consider the case where $C_{1}$ and $C_{2}$ are homeomorphic and let $k$ be any homeomorphism from $X$ onto $X$ such that $k\left[C_{1}\right]=C_{2}, k\left[C_{2}\right]=C_{1}$ and $k \circ k$ is the identity map. Again, let $\mathscr{F}$ be any countable collection of continuous selfmaps of $X$ and define $\mathscr{F}_{j}, 1 \leqq j \leqq 4$ just as before. Now let $\mathscr{F}_{2}^{*}=\left\{k \circ f: f \in \mathscr{F}_{2}\right\}$ and let $\mathscr{F}_{4}^{*}=$ $\left\{k \circ f: f \in \mathscr{F}_{4}\right\}$. Next, use the family $\mathscr{F}_{1} \cup \mathscr{F}_{2}^{*}$ to produce a continuous selfmap $h_{1}$ just as the map $g_{5}$ was produced in the proof of Theorem (2.4) and, similarly, use $\overline{\mathscr{F}}_{3} \cup \mathscr{F}_{4}^{*}$ to produce another continuous selfmap analogous to $g_{5}$. Again, one shows, just as in the proof of that theorem that $\mathscr{F}_{1} \cup \mathscr{F}_{2}^{*} \subseteq\left\langle\left\langle g_{1}, g_{2}, g_{3}, g_{4}, h_{1}\right\rangle\right\rangle$ and $\mathscr{F}_{3} \cup \mathscr{F}_{3}^{*} \subseteq$ $\left\langle\left\langle g_{1}, g_{2}, g_{3}, g_{4}, h_{2}\right\rangle\right\rangle$. Since $k \circ k$ is the identity map, it follows that $\mathscr{F}_{2}=\left\{k \circ f: f \in \mathscr{F}_{2}^{*}\right\}$ and $\mathscr{F}_{4}=\left\{k \circ f: f \in \mathscr{F}_{4}^{*}\right\}$, and from this, it readily follows that $\mathscr{F} \subseteq$ $\left\langle\left\langle g_{1}, g_{2}, g_{3}, g_{4}, h_{1}, h_{2}, k\right\rangle\right\rangle$ and, consequently, $S(S(X)) \leqq 7$ in this case.

\section{The density index}

As we mentioned in the introduction, it will be assumed throughout this section that topology on $S(X)$ is the compact-open topology. We recall that a basis for this topology is formed by taking all sets of the form $\langle K, G\rangle$ where $K$ is a compact subset of $X, G$ is an open subset of $X$ and $\langle K, G\rangle=\{f \in S(X): f[K] \subseteq G\}$. In the event $K$ consists of a single point $p$, we will use the notation $\langle p, G\rangle$ rather than the more cumbersome $\langle\{p\}, G\rangle$.

Definition 3.1. Let $A$ be a subset of a topological space $X . S(X)$ is said to be doubly transitive on $A$ if for points $a, b, x, y \in A$ with $x \neq y$, there exists an $f \in S(X)$ such that $f(a)=x$ and $f(b)=y$.

Theorem 3.2. Let $X$ be a compact disconnected space with a finite number of components at least one of which contains more than one more point. Suppose also that $S(X)$ is doubly transitive on each of its components. Then $D(S(X)) \geqq 3$.

Proof. We have already observed that $D(S(X)) \geqq 2$ if $X$ has more than one point. Suppose, in this case that $D(S(X))=2$. Then there are two continuous selfmaps $f$ and $g$ of $S(X)$ such that $\langle\langle f, g\rangle\rangle$ is dense in $S(X)$. Suppose that neither map is surjective. Let $G=X \backslash \operatorname{Ran} f$ and $H=X \backslash \operatorname{Ran} g$ (where Ran denotes the range of a function) and choose $p \in G$ and $q \in H$. Then $\langle p, G\rangle \cap\langle q, H\rangle \neq \varnothing$ since $S(X)$ is doubly transitive on its components but it does not contain an element from the subsemigroup $\langle\langle f, g\rangle\rangle$. Thus, at least one of the maps is surjective and we may well assume that is $f$. Denote the components of $X$ by $\left\{C_{j}\right\}_{j=1}^{N}$ and suppose Rang doesn't intersect one of those components, say $C_{k}$. Let $C_{t}$ be a component with more than one point and let $H$ be a proper nonempty open subset of $C_{t}$. Next, let $V=n\left\langle C_{j}, G_{j}\right\rangle_{j=1}^{N}$ where $G_{j}=C_{j}$ for $j \neq t$, $G_{t}=H . V$ is a nonempty open subset of $S(X)$ and therefore contains an element $h$ from $\langle\langle f, g\rangle\rangle$. Now $h$ cannot contain $g$ as a factor since $(\operatorname{Ran} h) \cap C_{j} \neq \varnothing$ for all $j$. Thus $h=f^{n}$ for some positive integer $n$. But this means $h$ is surjective and this is a contradiction since $h$ maps each $C_{j}$ into $C_{j}$ and actually maps $C_{t}$ properly into $C_{t}$. Therefore, $(\operatorname{Ran} g) \cap C_{j} \neq \varnothing$ for each $j$. But it readily follows from this that $(\operatorname{Ran} h) \cap$ 
$C_{j} \neq \varnothing$ for each $j$ where $h$ is any element in $\langle\langle f, g\rangle\rangle$. Thus $\langle\langle f, g\rangle\rangle \cap\left\langle X, C_{1}\right\rangle=\varnothing$ and again we have a contradiction. Consequently, we see that two elements of $S(X)$ cannot possibly generate a dense subsemigroup and we conclude that $D(S(X)) \geqq 3$.

We note that if $S(X)$ is separable then $D(S(X)) \leqq C(S(X))$. This is immediate if $C(S(X))=\infty$. On the other hand, if $C(S(X))=N$ for some positive integer $N$, simply choose a countable dense subset $\mathscr{F}$ of $S(X)$ and we are assured that this is contained in a subsemigroup with $N$ generators. Furthermore, it follows from the theorem in [4] that if $X$ is second countable and regular, then $S(X)$ is, in fact, hereditarily separable. These observations together with Theorem (2.4) immediately yield:

Corollary 3.3. Let $X$ consist of a finite number of components, each of which is a compact $N$-dimensional subspace of Euclidean $N$-space and has the internal extension property. Then $D(S(X))$ is finite.

Corollary 3.4. Let $X$ consist of two components, each of which is a compact $N$-dimensional subspace of Euclidean $N$-space and has the internal extension property. Then $3 \leqq D(S(X)) \leqq 8$ and if the two components are homeomorphic then $D(S(X)) \leqq 7$.

Proof. This is a consequence of the observations made preceding Corollary 3.2 and Theorems 2.5 and 3.1 .

Some closing remarks. Anytime we are able to show that $D(S(X)) \geqq N$ for separable $S(X)$ we can immediately conclude that $C(S(X)) \geqq N$ as well. Nevertheless, we chose in Theorem 2.2 to prove directly that $C(S(X)) \geqq 3$ without reference to $D(S(X))$ because we were able to get the result for a much more general class of spaces.

\section{REFERENCES}

1. H. Cook and W. T. Ingram, Obtaining AR-like continua as inverse limits with only two bonding maps, Glas. Mat. Ser. III 4 (1969), 309-312.

2. W. Hurewicz and H. Wallman, Dimension Theory (Princeton University Press, Princeton, 1941).

3. K. D. MAGill, JR., Some Open Problems and Directions for Further Research in Semigroups of Continuous Selfmaps (Univ. Alg. and Apps., Banach Center Pub., PWN-Polish Sci. Pub., Warsaw 9, 1982), 439-454.

4. E. A. Michael, On a theorem of Rudin and Klee, Proc. Amer. Math. Soc. 12 (1961), 921.

5. S. Subbiah, Some finitely generated subsemigroups of $S(X)$, Fund. Math. 86 (1975), 221-231.

6. S. Subbian, The compact-open topology for semigroups of continuous selfmaps, Semigroup Forum 35 (1987), 29-33.

106 Diefendorf Hall

SUNY AT BUFFaLO

Buffalo, New York 14214-3093

U.S.A. 